\title{
REVERSIBLE PHOTOCHEMICAL \\ REACTIONS OF \\ THYMINES HAVING LONG ALKYL CHAIN
}

\author{
Yoshiaki Inaki, Yuhua Wang, Takao Saito, and Kiichi Takemoto
}

Faculty of Engineering, Osaka University,

Yamadaoka 2-1, Suita, Osaka 565, Japan

Thymine bases convert to photodimers upon irradiation to UV light near the $\lambda$ max $(280 \mathrm{~nm})$. The photodimerization is a reversible reaction and the photodimers split to afford the original thymines very efficiently upon irradiation at a shorter wavelengths $(240 \mathrm{~nm})$.<smiles>Cc1cn(P)c(=O)[nH]c1=O</smiles>

Thymine Compound

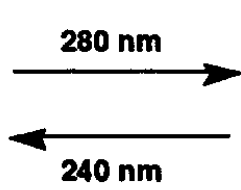

$240 \mathrm{~nm}$

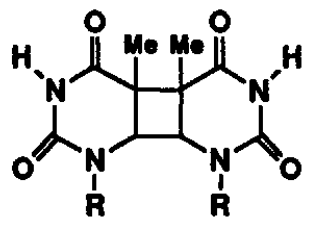

Photodimer

The reversible and thermally stable photochromic system of thymine can be applied to reversible photorecording system, where recording by UV irradiation at $280 \mathrm{~nm}$, reading at $270 \mathrm{~nm}$, and rewriting after irradiation at $240 \mathrm{~nm}$ can be carried out. The photodimerization reaction of thymine causes decrease of absorbance at around 270 $\mathrm{nm}$, which increases again by splitting of the photodimer. For high sensitivity and high efficiency of the reversible photodimerization reactions, orientation and aggregation of the thymine bases should be important factors [1]. This paper deals with the effect of chain length on the photochemical reactions of the thymine derivatives.

The thymine derivatives having long alkyl substituents gave a clear film by casting onto quartz plate. Figure 1 is the data of 1-myristylthymine (1) in film (glassy) state. Irradiation of $280 \mathrm{~nm}$ UV light caused the dimerization of thymine base and absorbance decreased, irradiating $240 \mathrm{~nm}$ UV light caused the splitting reaction of photodimer, then the absorbance increased. This compound shows wonderful reactivity, and perfect reversibility on photoreversible dimerization and splitting reactions by ir- 
radiating UV light at $280 \mathrm{~nm}$ and $240 \mathrm{~nm}$, respectively (Fig. 1A). Photodimerization and splitting reactions were fast as compared to the reactions in solution, and were repeated quantitatively for repeated runs. The rates of the photodimerization (Fig. 1B) have a turning point both for the firs and the second runs of photodimerization.
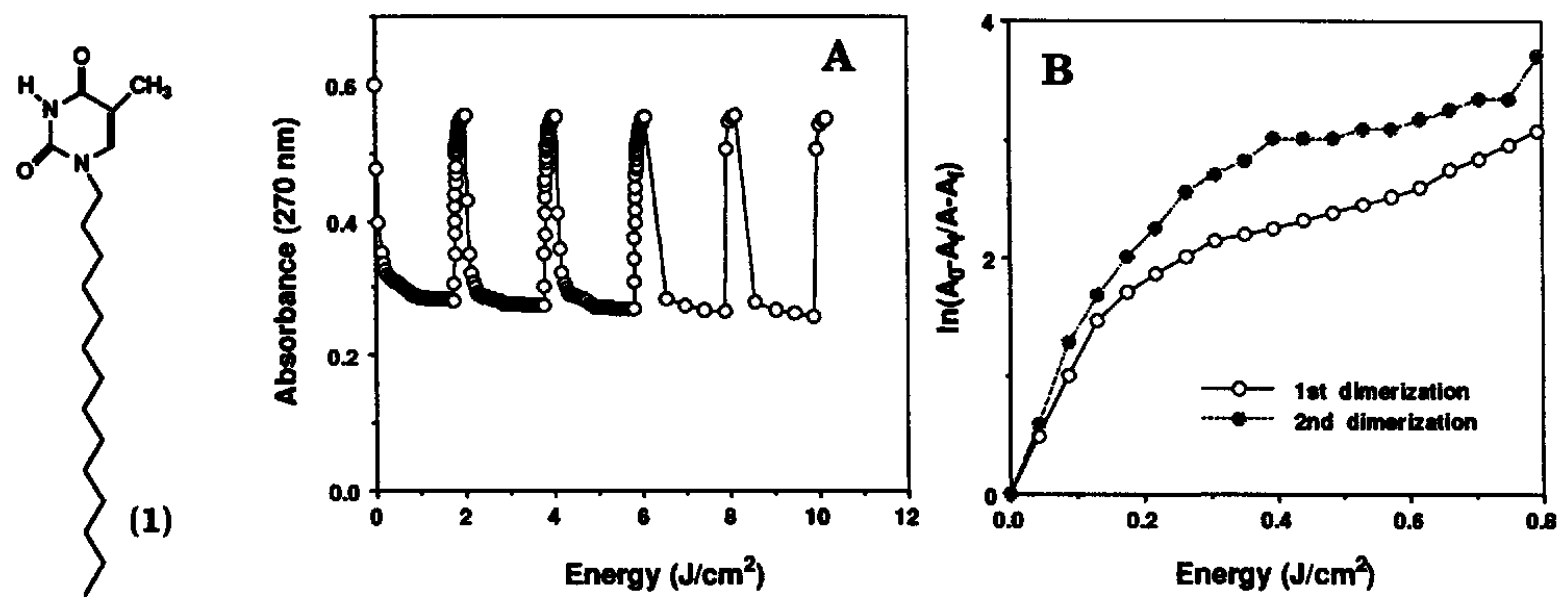

Figure 1. Reversible photodimerizations of 1-myristylthymine (1) in film state (A), and the rate of the photodimerization reactions (B).

Photodimerizations were also studied for the ester derivatives having long alkyl chain (n-decyl alcohol) (2). Figure 2 shows the reversible photodimerization reactions (A), and the rate of the photodimerization reactions (B). In this case, however, the reversibility was not good (Fig. 2A), and the second run of photodimerization did not have the turning point that was shown in the first run of photodimerization (Fig. 2B). The difference of the photochemical reactions between the alkyl and the ester compounds may be caused by the difference of the orientation of the compounds.
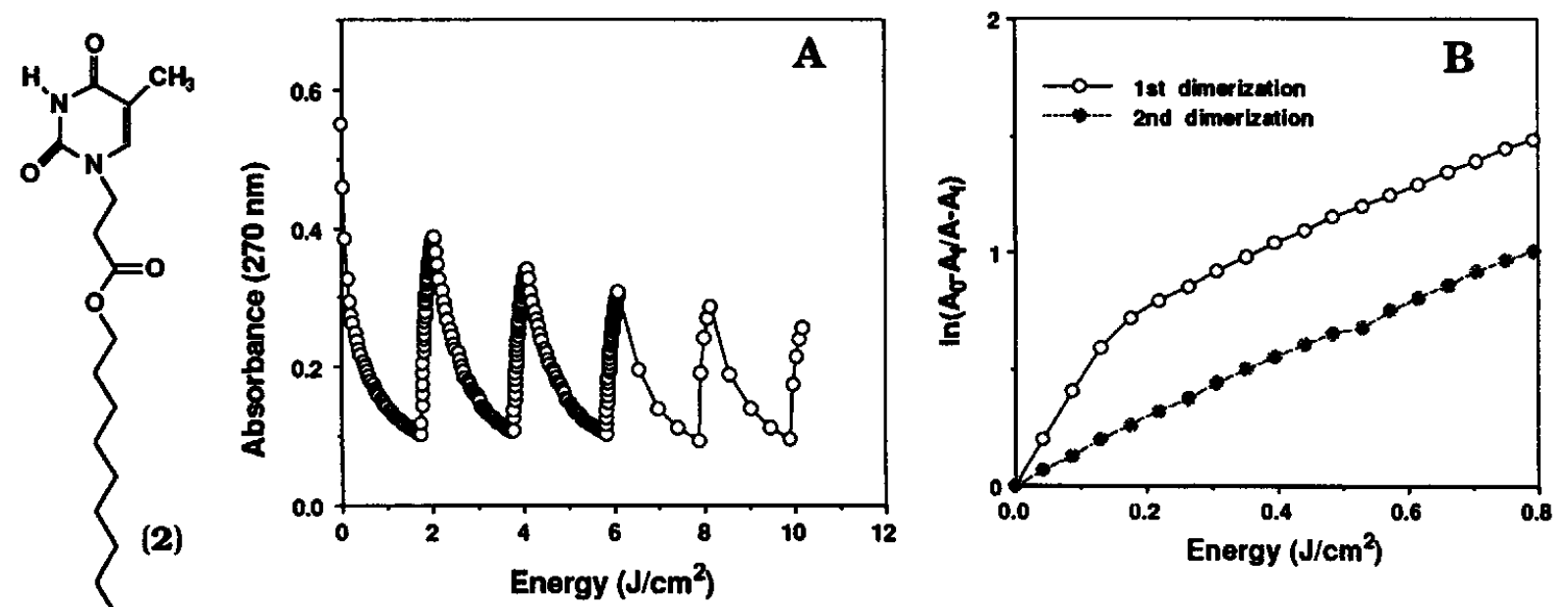

Figure 2. Reversible photodimerizations of 1-n-decylthymine derivative (2) in film state (A), and the rate of the photodimerization reactions (B).

[1] Y. Inaki, Y. Wang, and K. Takemoto, J. Photopolym. Sci. Technol., 4 (1991) 259. 